14

\title{
Температурная эволюция взаимодействия релаксационных процессов с локальной динамикой на терагерцевых частотах в полимерах с водородными связями
}

\author{
(C) B.A. РЫжов \\ Физико-технический институт им. А.Ф. Иофффе РАН, \\ Санкт-Петербург, Россия \\ E-mail: v.ryzhov@mail.ioffe.ru
}

Поступила в Редакцию 8 декабря 2021 г.

В окончательной редакции 8 декабря 2021 r.

Принята к публикации 15 декабря 2021 г.

\begin{abstract}
На терагерцевых частотах крутильно-колебательное движение связано с диэлектрической релаксацией в неупорядоченных твердых телах с водородными связями. Взаимодействие между этими процессами ещё мало изучено, особенно при температурах ниже температуры стеклования, что особенно существенно для молекулярной подвижности в полимерах. Нами изучены полимеры с водородными связями (полиамид-6 и поливинилхлорид) при температурах от 90 до $400 \mathrm{~K}$ в диапазоне $0.25-4 \mathrm{THz}$ с использованием дальней ИК-спектроскопии. Три общих особенности наблюдались в спектре диэлектрических потерь $\varepsilon^{\prime \prime}(v)$ : а) при температурах значительно ниже температуры стеклования $T_{g}$ эти потери представлены низкочастотным крылом пика поглощения, обусловленного либрацией мономерных звеньев полимеров; б) при $0.7 T_{g}<T<T_{g}$ наблюдаются дополнительные температурно-зависимые потери, которые могут быть связаны с проявлением вторичных релаксационных процессов; с) при температурах выше $T_{g}$ преобладающим вкладом в терагерцевые потери становятся первичные процессы $\alpha$-релаксакции. Полученные результаты показывают, что эволюция терагерцевых потерь с температурой вызвана изменением структуры водородных связей, которое, по-видимому, является универсальным для систем с подобными межмолекулярными взаимодействиями.
\end{abstract}

Ключевые слова: полимеры, терагерцевые ИК-спектры, температурная зависимость, $\alpha$ - и $\beta$-релаксации, водородная связь.

DOI: 10.21883/FTT.2022.04.52190.255

\section{1. Введение}

Стеклообразные материалы с межмолекулярными водородными связями, в том числе, полимеры, обладают интригующими физическими свойствами, давно являющимися предметом интенсивных исследований как экспериментальных, так и теоретических [1,2]. Диэлектрические, рамановские и терагерцевые ИК-спектры демонстрируют поразительное сходство проявления молекулярной динамики для широкого круга таких неупорядоченных систем [3]. На низких частотах диэлектрическая спектроскопия фиксирует проявление первичной $\alpha$ - и вторичной $\beta$-релаксации, а также существование более быстрых релаксаций на границе между радиои терагерцевыми частотами [4]. В терагерцевом диапазоне частот рамановская спектроскопия и спектроскопия нейтронного рассеяния показывают, что все стекла и полимеры проявляют здесь избыточные колебания, так называемый бозонный пик (БП) [5]. Эти дополнительные (по сравнению с моделью Дебая) колебательные моды, типа коррелированных крутильных колебаний в цепочке из нескольких тетраэдров в аморфном кварце, связывают с наличием в неупорядоченных средах среднего порядка [6]. На более высоких частотах взаимодействие излучения дальнего ИК-диапазона со стеклообразным твердым телом описывается в терминах плотности колебательных состояний (ПКС), которая формирует полосу либрационных колебаний его дипольных группировок [7].

В то время как взаимодействие между переориентационным движением и различными релаксационными процессами изучено довольно подробно [8], о взаимосвязи этой крупномасштабной подвижности с локальной крутильно-колебательной (либрационной) динамикой мало что известно.

В этой работе исследуется, с помощью ИК-спектроскопии в терагерцевом диапазоне, происхождение экспериментально наблюдаемых диэлектрических потерь в полимерах полиамиде-6 и поливинилхлориде на частотах от 0.25 до $3 \mathrm{THz}$ в температурном интервале от 90 до $400 \mathrm{~K}$, включающем температуру стеклования $T_{g}$. В литературе доступны лишь ограниченные данные о диэлектрических потерях на частотах близких к терагерцевым, полученные для полимеров и стекол другими методами [9].

\section{2. Методика}

Опыты проводились на пленках блочного атактического поливинилхлорида (ПВХ) и полиамида-6 
(ПА-6) со среднечисловыми молекулярными весами $M_{n}$ $\sim 5 \cdot 10^{5} \mathrm{~g} / \mathrm{mol}$ и $\sim 3 \cdot 10^{5} \mathrm{~g} / \mathrm{mol}$, соответственно, толщиной $\sim 100 \mu \mathrm{m}$, полученных прессованием при $150^{\circ} \mathrm{C}$ и выдержкой в течение $3-5 \mathrm{~h}$ при $110^{\circ} \mathrm{C}$. Спектры в дальнем инфракрасном диапазоне (FIR-спектры) регистрировались на двух спектрометрах: в диапазоне $8-50 \mathrm{~cm}^{-1}$ - на спектрометре ЛГУ [10], модернизированном с помощью приемника ОАП-7 и новой системы фильтрации; в диапазоне $50-150 \mathrm{~cm}^{-1}$ - на спектрометре FIS-21 Hitachi. Спектры записывались с разрешением $1-2 \mathrm{~cm}^{-1}$ при отношении сигнал/шум порядка 100. Погрешность измерения частоты составляла $2-3 \mathrm{~cm}^{-1}$. Погрешность измерения коэффициента поглощения $k(v)=(t)^{-1} \ln \left(I_{0} / I\right)$, где $I_{0}$ и $I$ - интенсивности падающего и прошедшего через образец толщиной $\mathrm{t}$ излучения соответственно, составляла 5-10\%. Для измерения спектров в температурном интервале от 90 до $400 \mathrm{~K}$ использовалась кювета-криостат с окнами из кристаллического кварца. FIR-спектры в интервале от комнатной температуры до $400 \mathrm{~K}$ получали, используя термостатированную кювету с сапфировыми окнами. Теплоизоляция в обоих случаях достигалась размещением кювет в вакууме. Температура образца измерялась термопарой медь-константан, ее стабильность была не ниже $\pm 2^{\circ} \mathrm{C}$. В интервале от 8 до $50 \mathrm{~cm}^{-1}$ для каждой температуры проводилось до пяти параллельных измерений.

Для того чтобы можно было сравнивать терагерцевые ИК-спектры со спектрами диэлектрических потерь $\varepsilon^{\prime \prime}(v)$, коэффициент поглощения $k(v)$ и показатель преломления $n(v)$ преобразуются по методу, предложенному в работе [11], в действительную и мнимую части диэлектрической функции:

$$
\begin{gathered}
\hat{\varepsilon}(v)=\varepsilon^{\prime}(v)+i \varepsilon^{\prime \prime}(v)=\hat{n}(v)^{2}, \\
\hat{n}(v)=n(v)+i \kappa(v), \\
\kappa(v)=(k(v) / v)(c / 4 \pi), \\
\varepsilon^{\prime}(v)=n(v)^{2}-\kappa(v)^{2}, \\
\varepsilon^{\prime \prime}(v)=2 n(v) \kappa(v)=(k(v) / v)(n(v) c / 2 \pi),
\end{gathered}
$$

где $\hat{\varepsilon}(v)$ - комплексная диэлектрическая функция, $\hat{n}(v)$ - комплексный показатель преломления, $n(v)-$ его действительная часть, дисперсия которой рассчитывается по соотношению Крамерса-Кронига с использованием данных по температурной зависимости коэффициента поглощения $k(v), \kappa(v)$ - коэффициент экстинкции, $c-$ скорость света в вакууме, $\varepsilon^{\prime}-$ диэлектрическая проницаемость, $\varepsilon^{\prime \prime}$ - диэлектрические потери. Поскольку $n(v)$ можно считать постоянной величиной в исследованном интервале частот $[12,13]$, $\varepsilon^{\prime \prime}(v) \approx$ const $\cdot k(v) / v$.

\section{3. Результаты и их обсуждение}

На рис. 1, 2 представлены в логарифмическом масштабе спектры диэлектрических потерь $\varepsilon^{\prime \prime}(v)$ ПВХ и ПА-6 в терагерцевом ИК-диапазоне при температурах от 90 до $400 \mathrm{~K}$.

Видно, что диэлектрические потери при низких температурах практически не зависят от температуры и

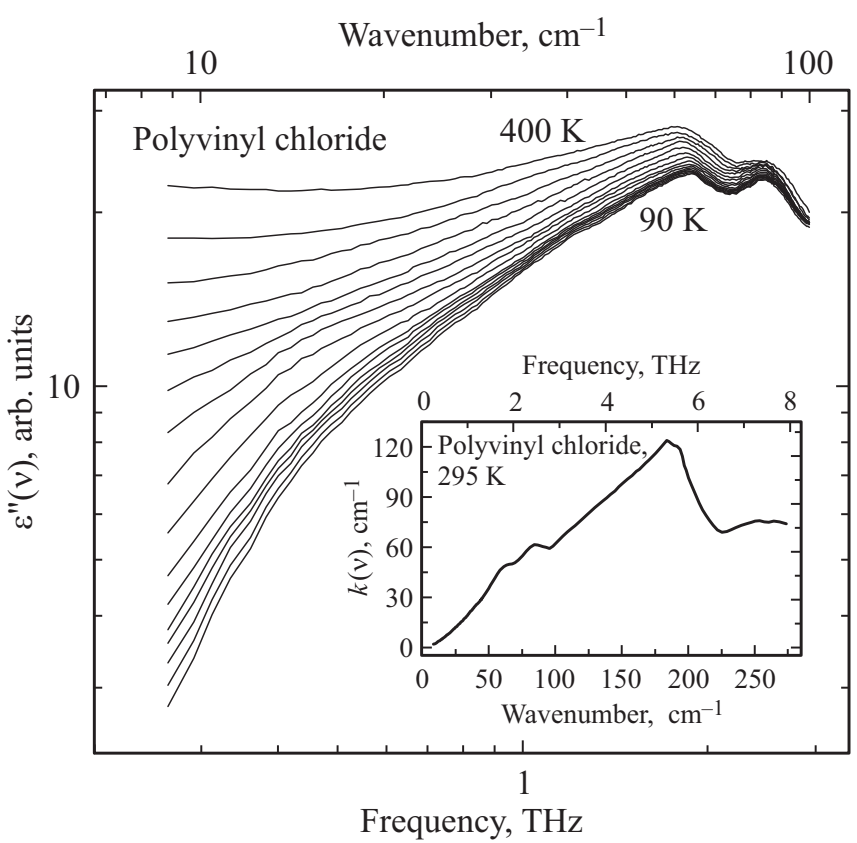

Рис. 1. Диэлектрические потери $\varepsilon^{\prime \prime}(v)$ ПВХ в интервале частот $0.25-3 \mathrm{THz}$ при температурах, изменяющихся в диапазоне $90-400 \mathrm{~K}$ с шагом $25 \mathrm{~K}$. На вставке приведен FIR-спектр $k(v)$ ПВХ при $295 \mathrm{~K}$ в области $8-275 \mathrm{~cm}^{-1}$, включающей исследованный диапазон терагерцевых частот.

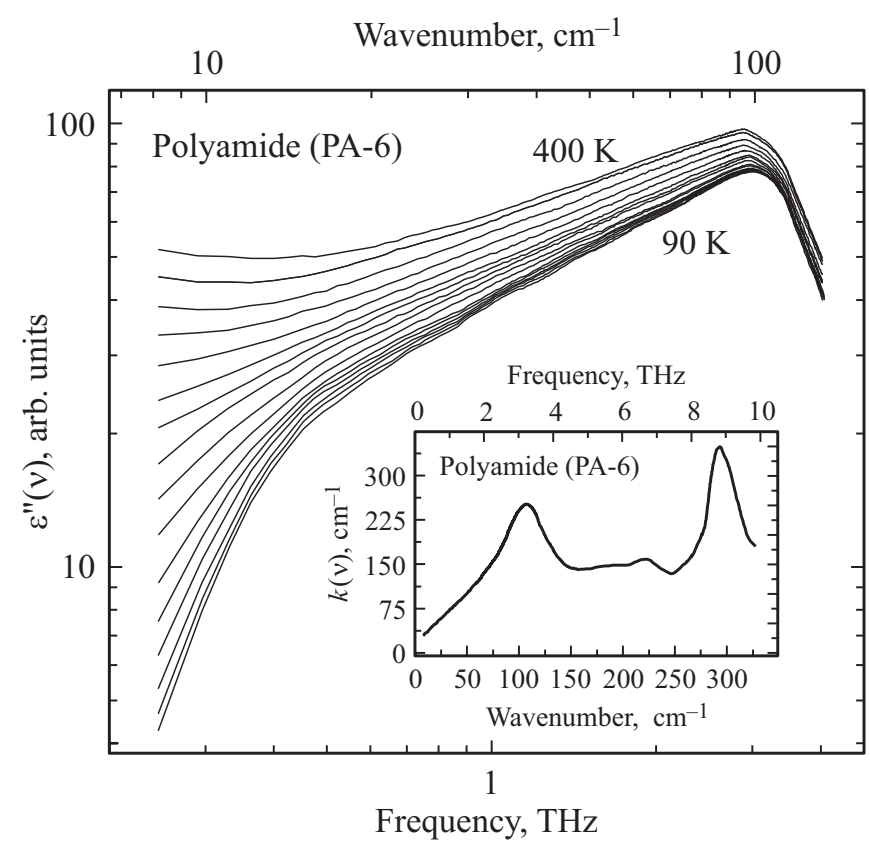

Рис. 2. Диэлектрические потери $\varepsilon^{\prime \prime}(v)$ ПА-6 в интервале частот $0.25-4 \mathrm{THz}$ при температурах, изменяющихся в диапазоне $90-400 \mathrm{~K}$ с шагом $25 \mathrm{~K}$. На вставке приведен FIR-спектр $k(v)$ ПА-6 при $295 \mathrm{~K}$ в области $8-325 \mathrm{~cm}^{-1}$, включающей исследованный диапазон терагерцевых частот. 
представляют собой низкочастотное крыло дублетной полосы поглощения с максимумами при 64 и $90 \mathrm{~cm}^{-1}$ в FIR-спектре ПВХ, которую относят к трансляционному движению на слабых водородных связях (ВС), типа $\mathrm{CH}$. . CCl, групп $\mathrm{CH}_{2} \mathrm{CCl}$ в конформационно различающихся цепях [14] (см. фрагмент структурной формулы).

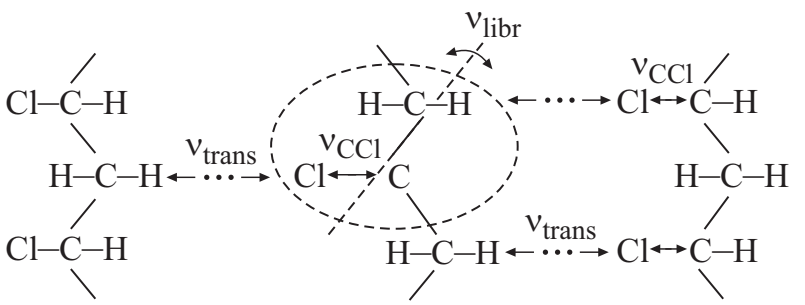

Диэлектрические потери при низких температурах в спектре ПА-6 представляют собой низкочастотное крыло полосы поглощения при $105 \mathrm{~cm}^{-1}$, которую относят к трансляционному движению на водородных связях группы CONH [15] (см. фрагмент структурной формулы).

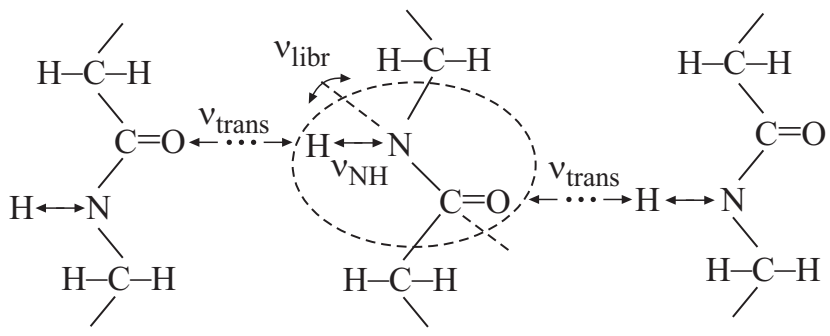

C повышением температуры эти высокочастотные пики в спектрах диэлектрических потерь ПВХ и ПА-6 растут по интенсивности и смещаются на $3-4 \mathrm{~cm}^{-1}$ к низким частотам в связи с ослаблением ВС и увеличением ангармонизма колебаний. Однако при этом интенсивность низкочастотного крыла полосы увеличивается существенно сильнее, чем высокочастотного, особенно на частотах ниже БП, который в использованных на рис. 1, 2 координатах выглядит как перегиб на спектральных кривых при 0.43 и $0.54 \mathrm{THz}$, соответственно.

Этот наблюдаемый рост с температурой диэлектрических потерь может быть связан с проявлением первичной диэлектрической релаксации, пик которой находится за пределами доступного для измерений диапазона частот при низких температурах и быстро смещается при температуре выше $T_{g}$ к терагерцевым частотам.

Очевидно, что проявление релаксационного вклада на терагерцевых частотах также обусловлено изменением межмолекулярных взаимодействий (в данном случае $\mathrm{BC})$, поскольку известно, что связанные ОН-группы в системах с ВС становятся свободными при температуре выше $T_{g}$ [16], увеличивая тем самым молекулярную подвижность.

Тот факт, что спектральные изменения с температурой на терагерцевых частотах значительнее, чем на частотах проявления крутильно-колебательного движения, позволяет использовать этот диапазон для получения информации о взаимосвязи и взаимовлиянии локальной
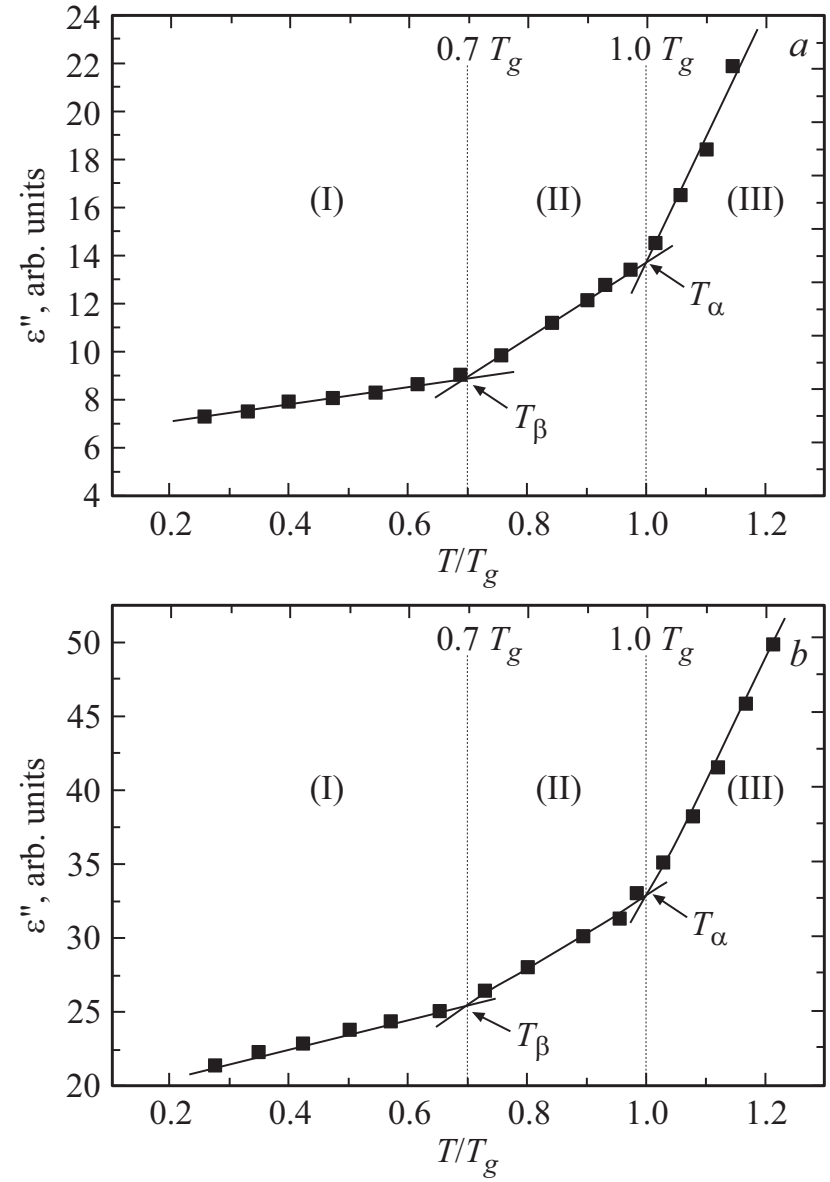

Рис. 3. Диэлектрические потери $\varepsilon^{\prime \prime}\left(T / T_{g}\right)$ на частотах $0.43 \mathrm{THz}$ для поливинилхлорида $(a)$ и $v=0.54 \mathrm{THz}$ для полиамида ПА-6 $(b)$. Сплошные линии представляют линейную аппроксимацию зависимости $\varepsilon^{\prime \prime}\left(T / T_{g}\right)$ в режимах (I), (II) и (III). $T_{\beta}$ и $T_{\alpha}$ - точки пересечения линейной аппроксимации (I) с (II) и (II) с (III), соответственно.

и крупномасштабной (сегментальной) динамик. Важной представляется также информация о том, каким образом система переходит от колебательной к релаксационной подвижности, приводящей, в итоге, к структурным изменениям.

Графики на рис. 3, $a, b$ иллюстрируют температурную эволюцию диэлектрических потерь в исследованном терагерцевом диапазоне на частоте, близкой к частоте БП: при $0.43 \mathrm{THz}\left(14.3 \mathrm{~cm}^{-1}\right)$ для ПВХ и $0.54 \mathrm{THz}$ $\left(18 \mathrm{~cm}^{-1}\right)$ для ПА-6. На рисунках представлены зависимости $\varepsilon^{\prime \prime}\left(T / T_{g}\right)$. Масштабированная температура $T / T_{g}$ по абсциссе использовалась для того, чтобы можно было сравнить общие характеристики стеклообразного состояния этих двух полимеров.

Из графиков видно, что в том и другом случае имеются, в основном, три режима температурной зависимости диэлектрических потерь: (I) практически температурнонезависимые потери при температурах далеких от температуры стеклования, (II) слабое увеличение потерь при приближении к температуре стеклования и (III 
Температуры стеклования и $\beta$-перехода, а также коэффициенты $B$ линейной аппроксимации диэлектрических потерь $\varepsilon^{\prime \prime}\left(T / T_{g}\right)=A+B T / T_{g}$ на разных участках зависимости $\varepsilon^{\prime \prime}\left(T / T_{g}\right)$, см. рис. $3, a, b^{*}$

\begin{tabular}{l|c|c|c|c|c|c|c|c}
\hline Полимер & $T_{g}, \mathrm{~K}$ & $T_{\beta}, \mathrm{K}$ & $T_{\beta} / T_{g}$ & $B_{\text {I }}$ & $B_{\text {II }}$ & $B_{\text {III }}$ & $v_{\mathrm{BP}, \mathrm{cm}^{-1}}$ & тип ВС \\
\hline ПВХ & 350 & $230-250$ & $0.66-0.72$ & 4 & 10 & 52 & $14(13)$ & $\mathrm{CH} \ldots \mathrm{ClC}$ \\
ПА-6 & $330-350$ & 220 & $0.68-0.63$ & 6 & 24 & 82 & $18(20)$ & $\mathrm{NH} \ldots \mathrm{OC}$
\end{tabular}

* Примечание. $T_{g}$ и $T_{\beta}$ - температуры стеклования и $\beta$-перехода, соответственно, взятые из литературы $[17] ; B_{\mathrm{I}}, B_{\mathrm{II}}$ и $B_{\text {III }}$ - линейные коэффициенты, полученные из анализа диэлектрических потерь в режимах (I), (II) и (III), соответственно; v числа в скобках взяты из работ [22] для ПВХ и [23] для ПА-6.

резкий рост потерь при $T>T_{g}$. При этом как в случае ПВХ, так и в случае ПА-6, переход от режима (I) к режиму (II) и от режима (II) к режиму (III) происходит при $T_{1}=0.7 T_{g}$ и $T_{2}=1.0 T_{g}$.

Температура стеклования (при $\alpha$-переходе) $T_{g}=350 \mathrm{~K}$ для ПВХ и $330-350 \mathrm{~K}$ для ПА-6; значит для ПВХ $T_{1}=245 \mathrm{~K}$, а для ПА-6, $T_{1}=230-245 \mathrm{~K}$. Такие температуры близки к температуре $\beta$-перехода, связанного с локальной конформационной подвижностью в этих полимерах [17]. Именно с температуры $T_{\beta}$, помимо чисто колебательного вклада, в спектре диэлектрических потерь начинают проявляться и растут потери, обусловленные переориентационной подвижностью макромолекулы.

Резкий скачок потерь при $T_{g}$ - это вклад основного в полимерах релаксационного процесса: $\alpha$-перехода стеклования, пик которого при температуре выше $T_{g}$ смещается к высоким частотам и его высокочастотное крыло попадает в терагерцевую область. Вполне очевидно, что проявление этого вклада на терагерцевых частотах также связано с изменением межмолекулярных взаимодействий (в данном случае ВС), поскольку, как уже отмечалось, связанные ОН-группы в системах с ВС становятся свободными при температуре выше $T_{g}$.

Рост релаксационного вклада при $T_{\beta}<T_{g}$, видимо, вызван не разрывом ВС, а лишь их ослаблением, однако достаточным, чтобы проявилась переориентационная подвижность в макромолекулах ПВХ и ПА-6r за счет увеличения с температурой амплитуд крутильноколебательных движений и накопления угловых колебательных смещений в цепи [18].

Из рис. 3, $a, b$ также видно, что некоторые незначительные температурные изменения в спектрах существуют и при температуре ниже $0.7 T_{g}$. Они могут быть вызваны другими вторичными релаксациями, например, такой как быстрая (fast- $\beta$ ) релаксация в полибутадиене [19], которую связывают с трансляционными колебаниями („rattling“) молекулы в ячейке, сформированной ее ближайшим окружением. Кроме того, небольшое красное смещение и уширение с температурой из-за ангармоничности самых низкочастотных колебательных полос (при $64 \mathrm{~cm}^{-1}$ в спектре ПВХ и при $105 \mathrm{~cm}^{-1}$ в спектре ПА-6, см. рис. 1,2), тоже дает вклад в режим (I).

В целом, единый сценарий температурного поведения терагерцевых спектров поливинилхлорида и полиамида6 показывает, что межмолекулярный, водородносвязывающий характер взаимодействий в этих полимерах играет одинаковую роль, как в первичной, так и во вторичной релаксации. Универсальность такого сценария следовало бы проверить в дальнейшем на других полимерах, где преобладающими являются ван-дер-ваальсовские взаимодействия.

В качестве уточнения обсуждаемой выше универсальности температурного поведения спектров, заметим, что температурные градиенты потерь спектров ПА-6 и ПВХ - коэффициенты $B$ линейной аппроксимации зависимости $\varepsilon^{\prime \prime}\left(T / T_{g}\right)=A+B T / T_{g}$ (см. таблицу) - всетаки разные: диэлектрические потери в спектре ПА-6 растут с температурой быстрее, чем в спектре ПВХ. Обнаруженный эффект, по-видимому, обусловлен тем, что макромолекулы ПВХ обладают повышенной по сравнению с ПА-6 жесткостью, которая определяется не только полярностью связи, но и коррелированностью движения соседних диполей. Энергия ВС равна $8.4 \mathrm{kcal} / \mathrm{mol}$ в ПА-6 [20] и $3.7 \mathrm{kcal} / \mathrm{mol}$ в ПВХ [21], а характеризующий равновесную жесткость полимерных цепей сегмент Куна для ПВХ включает двенадцать мономерных звеньев, тогда как для ПА-6 - всего два-три звена [17]. Можно предположить, что повышенная гибкость макромолекул ПА-6 позволяет сетке ВС в этом полимере более динамично реагировать на изменение температуры, чем в случае ПВХ.

Чувствительность описанного выше метода анализа к деталям температурно-зависимого поведения спектров свидетельствует о перспективности использования терагерцевого диапазона для изучения молекулярной динамики макромолекул.

\section{4. Заключение}

В целом, проведенный анализ диэлектрических потерь на терагерцевых частотах для двух полимеров, отличающихся типом ВС, при температурах выше и ниже $T_{g}$, показал ряд универсальных особенностей в их терагерцевых ИК-спектрах. При самых низких температурах эти потери представлены только низкочастотным крылом пика поглощения, обусловленного крутильноколебательным движением мономерных звеньев полимеров (при $64 \mathrm{~cm}^{-1}$ в спектре ПВХ и при $105 \mathrm{~cm}^{-1}$ в спектре ПА-6). До температур, близких к температуре $\beta$-перехода в ПВХ и ПА-6, равной $0.7 T_{g}$, они практически не зависят от температуры и, таким образом, рост диэлектрических потерь, начиная с $T=0.7 T_{g}$, может 
быть связан с проявлением на терагерцевых частотах вторичной релаксации. Следующий, более резкий скачок потерь происходит при $T=T_{g}$, когда в терагерцевый диапазон смещается высокочастотное крыло $\alpha$-процесса. При температурах выше $T_{g}$ релаксационные вклады становятся преобладающими в терагерцевых спектрах ПВХ и ПА-6. Поскольку релаксационная подвижность в полимерах в основном контролируется потенциальными барьерами межмолекулярных взаимодействий водородными связями в данном случае - проявление локального молекулярного движения при $T_{\beta}$ свидетельствует об их ослаблении. Резкое увеличение релаксационного вклада в спектры при повышении температуры до $T_{g}$ - проявление сегментальной подвижности - вызвано, по-видимому, не только ослаблением и разрывом ВC, но и изменением в системе водородных связей в исследованных полимерах. Это заключение подтверждает тот факт, что межмолекулярные взаимодействия играют важную роль в первичной и вторичной релаксациях, а также подчеркивает необходимость учета релаксационного вклада в терагерцевый диапазон ИК-спектра. Дальнейшие терагерцевые исследования на других объектах с ВС и без них необходимы для подтверждения универсальности взаимосвязи релаксационных процессов с низкочастотными крутильно-колебательными модами.

\section{Конфликт интересов}

Автор заявляет об отсутствии конфликта интересов.

\section{Список литературы}

[1] E. Kremer, A. Loidl. The Scaling of Relaxation Processes. Springer International Publishing AG, Leipzig (2018). 325 p.

[2] G. Biroli, J.P. Garahan. J. Chem. Phys. 138, 12 A301 (2013).

[3] E.P.J. Parrot, A. Zeitler. Appl. Spectrosc. 69, 1, 1 (2015).

[4] W. Götze. Complex Dynamics of Glass-Forming Liquids. Oxford University Press, N.Y. (2009). 362 p.

[5] V.N. Novikov, A.P. Sokolov, B. Stube, N.V. Surovtsev, E. Duval, A. Mermet. J. Chem. Phys. 107, 1057 (1997).

[6] S.D. Bembenek, B.B. Laird. J. Chem. Phys. 114, 2340 (2001).

[7] V.A. Bershtein, V.A. Ryzhov. Adv. Pol. Sci. 114, 43 (1994).

[8] K.L. Ngai. Relaxation and Diffusion in Complex Systems. Oxford University Press, N.Y. (2011). 425 p.

[9] S. Kastner, M. Köhler, V. Goncharov, P. Lunkenheimer, A. Loidl. J. Non-Cryst. Solids 357, 510 (2011).

[10] В.А. Рыжов, М.В. Тонков. В сб.: Молекулярная спектроскопия / Под ред. Г.С. Денисова. ЛГУ, Л. (1973). С. 108.

[11] L. Duvillaret, F. Garet, J.-L. Coutaz. IEEE J. Sel. Top. Quantum Electron. 2, 739 (1996).

[12] Y-S. Jin, G-J. Kim, S-G. Jeon. J. Korean Phys. Soc. 40, 513 (2006).

[13] S. Wietzke, C. Janden, M. Reuter, B.M. Ficsher, M. Koch, J. Mol. Struct. 1006, 41 (2011).

[14] A.V.R. Warrier, S. Krimm. Macromolecules 3, 709 (1970).

[15] W.F.X. Frank, H. Fiedler. Infrared Phys. 19, 481 (1979).

[16] A. Barkatt, C.A. Angell. J. Chem. Phys. 70, 901 (1979).

[17] В.А. Берштейн, В.М. Егоров. Дифференциальная сканирующая калориметрия в физикохимии полимеров. Химия, Л. (1990). 248 c.
[18] Ю.Я. Готлиб, А.А. Даринский, Ю.Е. Светлов. Физическая кинетикамакромолекул. Химия, Л. (1986). 272 с.

[19] R. Zorn, A. Alegria, A. Arbe, J. Colmenero, D. Richter, B. Frick. J. Non-Cryst. Solids 235-237, 169 (1998).

[20] N. Nagai, T. Imai, R. Fukasawa, K. Kato, K. Yamauchi. Appl. Phys. lett. 85, 4010 (2004).

[21] G. Wypych. PVC Degradation and Stabilization. ChemTec Publishing, Toronto (2008). $466 \mathrm{p}$.

[22] E. Duval, T. Achibat, A. Boukenter, B. Varrel, R. Calemczuk, B. Salce. J. Non-Cryst. Solids 190, 258 (1995).

[23] P. Papanek, J.E. Fischer, N.S. Murthy. Macromolecules 35, 10, 4175 (2002).

Редактор Е.Ю. Флегонтова 